\title{
El plástico biodegradable en el Perú: ¿una solución o un problema?
}

Biodegradable plastic in Peru: A solution, or a problem?

Roxana Diaz ${ }^{1 *} \odot$

1 Universidad Nacional Agraria La Molina. Lima, Perú.

\section{SOUTH} SUSTAINABILITY

Citar como: Diaz, R. (2021). «El plástico biodegradable en el Perú: ¿una solución o un problema?». South Sustainability, 2(2), e042. DOI: doi. org/10.21142/SS-0202-2021-pb004

Artículo recibido: 12/6/2020

Arbitrado por pares

Artículo aceptado: 31/12/2021

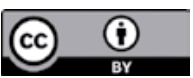

(c) La autora, 2021. Publicado por la Universidad Científica del Sur (Lima, Perú)

${ }^{*} E$-mail de correspondencia: roxanaydiaz@gmail.com

\section{RESUMEN}

La biodegradabilidad fue introducida por la legislación nacional como una alternativa al plástico convencional. Sin embargo, diversas publicaciones han concluido que la biodegradabilidad no es una solución para la problemática de la contaminación por plástico. Se analiza la legislación vigente, donde se encuentra un vacío sobre los plásticos biodegradables, por lo cual se recomienda la eliminación de la exoneración del impuesto al consumo de bolsas de plástico a las bolsas de plástico biodegradables, la obligatoriedad de que los bienes biodegradables sean también compostables, así como la incorporación de criterios objetivos para definir la biodegradabilidad y la compostabilidad.

Palabras clave: plástico, contaminación, biodegradable, compostable

\section{ABSTRACT}

Biodegradability was introduced through national legislation as an alternative to conventional plastic. However, a number of publications have concluded that biodegradability is not a solution to the problem of plastic pollution. This study analyzes current legislation, where there is a void when it comes to biodegradable plastics, in response to which the elimination is recommended of the exemption from the "consumption tax on plastic bags" for biodegradable plastic bags, the introduction of the obligation that biodegradable goods should also be compostable, as well as the incorporation of objective criteria to define biodegradability and compostability.

Keywords: plastic, pollution, biodegradable, compostable 


\section{Introducción}

En 2018, el plástico biodegradable fue introducido como una alternativa al plástico convencional mediante la Ley 30884, «Ley que regula el plástico de un solo uso y los recipientes o envases descartables». Aunque no se menciona textualmente en la norma, en el mercado se ha interpretado que se promueve el plástico biodegradable porque las bolsas de portabilidad de plástico que cuenten con certificados de biodegradabilidad son el único tipo de bien de plástico exonerados del impuesto al consumo de las bolsas de plástico.

En 2019, se aprobó el Reglamento de la Ley 30884 mediante el Decreto Supremo 006-2019-MINAM. La norma establece que la minimización en el uso de los bienes de plástico prevalece sobre su valorización. En ese sentido, se interpretaría que los bienes de plástico reutilizables se deben priorizar sobre los biodegradables.

Posteriormente, en diciembre de 2021, se aprobó el Reglamento Técnico sobre Bolsas de Plástico Biodegradable mediante el Decreto Supremo 025-2021-PRODUCE. Este dispositivo establece los requisitos técnicos y de etiquetado que deben cumplir las bolsas de plástico biodegradables: la identificación del polímero, la identificación de los constituyentes de la bolsa de plástico (incluyendo otros materiales diferentes al polímero, tintas y aditivos), la concentración mínima de sólidos volátiles, las concentraciones máximas de

Tabla 1. Estudios y reportes revisados y analizados.

\begin{tabular}{|c|c|c|c|}
\hline N..$^{\circ}$ & Año & Organización/ autor & Tema \\
\hline 1 & 2021 & $\begin{array}{l}\text { United Nations } \\
\text { Environment } \\
\text { Programme (UNEP) }\end{array}$ & $\begin{array}{c}\text { Drowning in plastics. Marine } \\
\text { litter and plastic waste vital } \\
\text { graphics }\end{array}$ \\
\hline 2 & 2021 & UNEP & $\begin{array}{l}\text { From pollution to solution: A } \\
\text { global assessment of marine } \\
\text { litter and plastic pollution }\end{array}$ \\
\hline 3 & 2020 & $\begin{array}{l}\text { Hann, S., Scholes, R., } \\
\text { Molteno, S., Hilton, } \\
\text { M., Faviono, E. y } \\
\text { Jakobsen Geest, L. / } \\
\text { Comisión Europea }\end{array}$ & $\begin{array}{c}\text { Relevance of biodegradable and } \\
\text { compostable consumer plastic } \\
\text { products and packaging in a } \\
\text { circular economy }\end{array}$ \\
\hline 4 & 2019 & $\begin{array}{l}\text { Napper, I. E. y } \\
\text { Thompson, R. C. }\end{array}$ & $\begin{array}{c}\text { Environmental deterioration } \\
\text { of biodegradable, oxo- } \\
\text { biodegradable, compostable, } \\
\text { and conventional plastic carrier } \\
\text { bags in the sea, soil, and open- } \\
\text { air over a 3-year period }\end{array}$ \\
\hline 5 & 2018 & Comisión Europea & $\begin{array}{c}\text { Una estrategia europea para } \\
\text { el plástico en una economía } \\
\text { circular }\end{array}$ \\
\hline 6 & 2016 & $\begin{array}{l}\text { The Ellen MacArthur } \\
\text { Fundation }\end{array}$ & $\begin{array}{l}\text { The new economic plastic: } \\
\text { Rethinking the future of plastics }\end{array}$ \\
\hline 7 & 2015 & UNEP & $\begin{array}{l}\text { Biodegradable plastics and } \\
\text { marine litter. Misconceptions, } \\
\text { concerns and impacts on } \\
\text { marine environments }\end{array}$ \\
\hline
\end{tabular}

metales y otras sustancias peligrosas, la determinación de biodegradabilidad, entre otros. Esta norma es el primer antecedente del país para definir objetivamente la biodegradabilidad de plásticos, aunque limitado a las bolsas de plástico con fines de portabilidad.

A la fecha, son diversos los estudios y reportes (ver tabla 1) que concluyen que el plástico biodegradable, e incluso compostable, no son la solución a la problemática del plástico. Sin embargo, a pesar de la evidencia técnica y científica y la aclaración de la normativa nacional, la preferencia de bienes de plástico etiquetados como «biodegradables» prevalece sobre el plástico reciclable y reutilizable en el país, especialmente en las bolsas de plástico con fines de portabilidad y en los envases para contener alimentos y bebidas.

\section{Enfoque y discusión}

La incertidumbre de los beneficios de los plásticos biodegradables fue identificada ya desde 2015, cuando el reporte «Biodegradable plastics and marine litter. Misconceptions, concerns and impacts on marine environments», del Programa de las Naciones Unidas para el Medio Ambiente, concluía que la adopción de bienes plásticos etiquetados como «biodegradables» no disminuyen significativamente la cantidad de plástico que ingresa al océano o el riesgo de impactos físicos y químicos en el mar (UNEP, 2015). Posteriormente, se publicaron estudios que mostraban casos de bolsas de plástico biodegradables que se mantenían casi completas incluso después de tres años en el medio marino (Napper et al., 2019). Recientemente, UNEP (2021a, b) señala que los plásticos etiquetados como biodegradables pueden tardar varios años en degradarse en los océanos y, como residuos, pueden presentar los mismos riesgos que los plásticos convencionales para las personas, la biodiversidad y el funcionamiento de los ecosistemas.

La Fundación Ellen MacArthur (2016) señaló que los plásticos biodegradables podrían convertirse en una buena alternativa siempre que estén acompañados de infraestructura apropiada para su recolección y valorización mediante el compostaje u otros similares. Asimismo, señala que, en una economía circular del plástico, se espera que estos bienes sean reutilizables/reciclables o biodegradables en condiciones de compostaje; por lo general, un producto no posee al mismo tiempo ambas características debido al actual desarrollo tecnológico y a que la infraestructura y la operación para su posterior valorización son diferenciadas para cada uno de estos tipos de bienes de plástico.

Asimismo, la Fundación Ellen MacArthur (2016) señala que, en la mayoría de los casos, el reciclaje es preferible al compostaje, ya que mantiene el material en la economía aportado a la circularidad del modelo, mientras que el compostaje descompone los envases/embalajes en compuestos de bajo valor económico. Sin embargo, en 
ciertas aplicaciones específicas, los envases/embalajes compostables podrían ser un mecanismo valioso para devolver nutrientes al suelo. Las aplicaciones más prometedoras son las que cumplen los siguientes criterios:

- Se utilizan para envasado y empaque de alimentos. En ambos casos son dispuestos como residuos orgánicos y valorizados mediante el compostaje. Por el contrario, envasar alimentos con plástico reciclable requiere lavar el material para su reciclaje.

El riesgo de que termine en flujos de reciclaje de plástico reutilizable y reciclable es mínimo, porque el plástico compostable disminuye la calidad del plástico reciclado.

Ante ello, se considera que los bienes de plástico biodegradables no son el sustituto por excelencia del plástico convencional. El tiempo de degradación, la composición del plástico, el método de valoración y el ambiente/infraestructura para degradación son cruciales para asegurar la biodegradación de este tipo de productos (UNEP, 2015; Comisión Europea, 2018).

Por ello, la aplicabilidad y determinación de la biodegradación debería ser revisada considerando el medio donde se desarrollará (UNEP, 2021b). Por ejemplo, en el compostaje industrial se pueden tomar como referencia las normas ISO 17088, EN 13432 y ASTM 6400 (UNEP, 2021b). Cabe señalar que el Reglamento Técnico sobre Bolsas de Plástico Biodegradable, si bien se basa en la norma ISO 17088, solo determina la biodegradabilidad en condiciones de laboratorio ya sea de compostaje o digestión anaeróbica, debido a que no incluyen los requisitos relacionados con requisitos de desintegración durante el compostaje y la ausencia de efectos adversos del compostaje en organismos terrestres.

En la misma línea, es importante también analizar si la biodegradación se puede aplicar en la práctica y escala (ISO 17088). Es decir, si operativamente se puede ejecutar dadas las condiciones particulares del país relacionadas, entre otras, con la centralización industrial, infraestructura, tecnología y gestión de residuos. Por ejemplo, Francia ha aprobado una regulación que solo considera biodegradables a los bienes de plástico compostables en el hogar (UNEP, 2021b).

Recientemente, Hann et al. (2020), en el reporte para la Comisión Europea sobre la relevancia de los productos y envases plásticos de consumo biodegradables y compostables en una economía circular, señalaron que la evidencia es débil a favor de cualquier beneficio agronómico particular asociado con material plástico compostable en compost o digestión y, por lo tanto, recomiendan que se prioricen productos reciclables sobre compostables. Asimismo, enfatizan en que los residuos plásticos compostables no degradados resultantes son un riesgo significativo que no puede cuantificarse actualmente. Al igual que lo menciona la fundación Ellen MacArthur, se han encontrado aplicaciones limitadas y muy específicas para el plástico compostable (Hann et al., 2020).

\section{Conclusiones}

La biodegradabilidad no es la solución a la problemática del plástico, requiere condiciones adecuadas para que ocurra, no se realiza en su totalidad y se le asocia a un consumo no responsable del plástico de un solo uso, porque el consumidor cree que al ser biodegradable no genera impacto. Además, puede contaminar la cadena del reciclaje del plástico.

En el mercado peruano hay mucha desinformación alrededor de la biodegradabilidad, por lo que se requiere establecer objetivos claros, ligar la biodegradabilidad a la compostabilidad y a la factibilidad de desarrollarla en relación con la infraestructura, la gestión de residuos y la tecnología en el país. Asimismo, se necesita realizar un análisis de trazabilidad de las evidencias de biodegradabilidad, especialmente para el caso de los certificados de conformidad y los informes de ensayo.

\section{Recomendaciones de política}

Dados los mayores impactos que beneficios del plástico biodegradable, se recomienda la eliminación de la exoneración del impuesto al consumo de bolsas de plástico a las bolsas de plástico biodegradables. Se recomienda asimismo la obligatoriedad de que los bienes biodegradables sean también compostables, así como la incorporación de criterios objetivos para definir la biodegradabilidad y la compostabilidad en plástico, incluyendo criterios vinculados al medio de biodegradación.

\section{Fuente de financiamiento}

Autofinanciado.

\section{Potenciales conflictos de interés}

No existe ningún conflicto de interés en este artículo.

\section{Referencias bibliográficas}

Comisión Europea. (2018). «Una estrategia europea para el plástico en una economía circular». Disponible en: https://www.europarl. europa.eu/doceo/document/TA-8-2018-0352_ES.html

Hann, S., Scholes, R., Molteno, S., Hilton, M., Faviono, E. y Jakobsen Geest, L. (2020). «Relevance of biodegradable and compostable consumer plastic products and packaging in a circular economy». European Commission. Disponible en: https://doi. org/10.2779/497376

ISO 17088:2021(en). (2021). «Plastics - Organic recycling - Specifications for compostable plastics». Disponible en: https://www.iso.org/obp/ ui/\#iso:std:iso:17088:ed-3:v1:en

Napper, I. E. y Thompson, R. C. (2019). «Environmental deterioration of biodegradable, oxo-biodegradable, compostable, and conventional plastic carrier bags in the sea, soil, and open-air over a 3-year period». Environmental Science \& Technology, 53(9), pp. 4775-4783. Disponible en: https://doi.org/10.1021/acs.est.8b06984 
The Ellen MacArthur Foundation. (2016). "The new economic plastic: Rethinking the future of plastics». Disponible en: https:// ellenmacarthurfoundation.org/the-new-plastics-economyrethinking-the-future-of-plastics

United Nations Environment Programme, UNEP. (2015). «Biodegradable plastics and marine litter. Misconceptions, concerns and impacts on marine environments. Nairobi». Disponible en: https://wedocs.unep.org/bitstream/handle/20.500.11822/7468/Biodegradable Plastics and Marine Litter Misconceptions, concerns_and_impacts_on_marine_environments-2015Biodegradab lePlasticsAndMarineLitter.pdf.pdf?sequence\%3D3
United Nations Environment Programme, UNEP. (2021a). Drowning in plastics. Marine litter and plastic waste vital graphics. Disponible en: https://wedocs.unep.org/xmlui/bitstream/ handle/20.500.11822/36964/VITGRAPH.pdf

United Nations Environment Programme, UNEP. (2021b). «From pollution to solution: A global assessment of marine litter and plastic pollution. Nairobi». Disponible en: https://www.unep.org/ resources/pollution-solution-global-assessment-marine-litter-andplastic-pollution 